\title{
Absence of a Metallicity Effect for Ultra-short-period Planets*
}

\author{
Joshua N. Winn ${ }^{1}$, Roberto Sanchis-Ojeda ${ }^{2}$, Leslie Rogers ${ }^{3}$, Erik A. Petigura ${ }^{4}$, Andrew W. Howard ${ }^{4}$, Howard Isaacson ${ }^{2}$, \\ Geoffrey W. Marcy ${ }^{2}$, Kevin C. Schlaufman ${ }^{5}$, Phillip Cargile ${ }^{6}$, and Leslie Hebb ${ }^{7}$ \\ ${ }^{1}$ Department of Astrophysical Sciences, Princeton University, 4 Ivy Lane, Princeton, NJ 08540, USA \\ ${ }^{2}$ Department of Astronomy, University of California, Berkeley, CA 94720, USA \\ ${ }^{3}$ Department of Astronomy \& Astrophysics, University of Chicago, 5640 South Ellis Avenue, Chicago, IL 60637, USA \\ ${ }^{4}$ Department of Astronomy, California Institute of Technology, Pasadena, CA 91125, USA \\ ${ }^{5}$ Department of Physics and Astronomy, Johns Hopkins University, Baltimore, MD 21218, USA \\ ${ }^{6}$ Harvard-Smithsonian Center for Astrophysics, 60 Garden Street, Cambridge, MA 02138, USA \\ ${ }^{7}$ Hobart and William Smith Colleges, Geneva, NY 14456, USA \\ Received 2017 April 1; revised 2017 June 17; accepted 2017 June 21; published 2017 July 20
}

\begin{abstract}
Ultra-short-period (USP) planets are a newly recognized class of planets with periods shorter than one day and radii smaller than about $2 R_{\oplus}$. It has been proposed that USP planets are the solid cores of hot Jupiters that have lost their gaseous envelopes due to photo-evaporation or Roche lobe overflow. We test this hypothesis by asking whether USP planets are associated with metal-rich stars, as has long been observed for hot Jupiters. We find the metallicity distributions of USP-planet and hot-Jupiter hosts to be significantly different $\left(p=3 \times 10^{-4}\right)$ based on Keck spectroscopy of Kepler stars. Evidently, the sample of USP planets is not dominated by the evaporated cores of hot Jupiters. The metallicity distribution of stars with USP planets is indistinguishable from that of stars with short-period planets with sizes between 2 and $4 R_{\oplus}$. Thus, it remains possible that the USP planets are the solid cores of formerly gaseous planets that are smaller than Neptune.
\end{abstract}

Key words: atmospheres - planetary systems - planets and satellites: detection - stars: abundances

\section{Introduction}

The discovery of planets with orbital periods shorter than one day, and comparable in size to that of the Earth, has sparked discussion about their origin and evolution. The first well-documented planets in this category were CoRoT-7b (Léger et al. 2009), Kepler-10b (Batalha et al. 2011), 55 Cnc e (Dawson \& Fabrycky 2010; Demory et al. 2011; Winn et al. 2011), and Kepler-78b (Sanchis-Ojeda et al. 2013). A sample of about 100 such planets was drawn together from Kepler data and analyzed by Sanchis-Ojeda et al. (2014). An independent Kepler survey was performed by Jackson et al. (2013), and new examples have since been discovered by Becker et al. (2015), Adams et al. (2016), and Vanderburg et al. (2016).

Among the hypotheses for the origin of these ultra-shortperiod (USP) planets is that they are the exposed solid cores of hot Jupiters that have formed through core accretion. As circumstantial evidence for a connection between USP planets and hot Jupiters, Sanchis-Ojeda et al. (2014) and Steffen \& Coughlin (2016) noted that these two categories of planets are both found around $\approx 0.5 \%$ of FGK stars. They also found that USP planets are almost always smaller than $2 R_{\oplus}$, putting them in or near the size range for which planets are thought to have a mainly rocky composition (Weiss \& Marcy 2014; Rogers 2015). They hypothesized that the most strongly irradiated hot Jupiters eventually lose their gaseous envelopes due to photo-evaporation or Roche lobe overflow (Valsecchi et al. 2014). This would leave behind a nearly naked core in a close-in orbit. Proving this hypothesis to be correct would confirm the core-accretion theory, and enable direct measurements of the size and mass

\footnotetext{
* Based on observations obtained at the W. M. Keck Observatory, which is operated jointly by the University of California and the California Institute of Technology. Keck time was granted by NASA, the University of California, the California Institute of Technology, and the University of Hawaii.
}

distribution of the rocky cores that nucleate the growth of giant planets.

However, there are other possibilities for the origin of the USP planets. They might represent the short-period extension of the distribution of close-in rocky planets that have either formed by core accretion in their current orbits (Chiang \& Laughlin 2013), or migrated inward from more distant orbits (Ida \& Lin 2004; Schlaufman et al. 2010; Terquem 2014). Another possibility is that the USP planets are the exposed remnants not of hot Jupiters, but of smaller gaseous planets with sizes between 2 and $4 R_{\oplus}$ (Lundkvist et al. 2016; Lee \& Chiang 2017).

Here we test for a connection between USP planets and hot Jupiters by comparing the metallicities of their host stars. Stars that host giant planets with orbital periods shorter than a few years have systematically higher metallicities than those of randomly chosen stars in the solar neighborhood (Gonzalez 1997; Santos et al. 2004; Fischer \& Valenti 2005). In contrast, the host stars of smaller planets show little, if any, association with high metallicity (Udry et al. 2006; Schlaufman \& Laughlin 2011; Sousa et al. 2011; Buchhave et al. 2012). ${ }^{8}$ If all USP planets are the cores of former hot Jupiters, we should observe similar metallicity distributions for the hosts of USP planets and hot Jupiters. If instead the progenitors of USP planets are gaseous planets less massive than hot Jupiters, or if they form in the same way as somewhat longer-period planets, then the stars with USP planets should have a metallicity distribution similar to that of shortperiod sub-Neptunes. The metallicity distribution of Kepler planet hosts has been investigated previously by Buchhave et al. (2012),

\footnotetext{
8 We note, though, that these studies focused on stars near solar metallicity, and that Zhu et al. (2016) have questioned some of the evidence. Furthermore, Wang \& Fischer (2015) found a metallicity effect for small planets, though not as strong as for giant planets, and Adibekyan et al. (2012) found that smallplanet hosts tend to be higher in $\alpha$-elements even if they are relatively poor in iron (the traditional metallicity indicator).
} 
Mann et al. (2013), Buchhave et al. (2014), Dong et al. (2014), Schlaufman (2015), Buchhave \& Latham (2015), Guo et al. (2017), and Mulders et al. (2016), but without special attention to USP planets. Our study focuses on USP planets, using the curated sample of Sanchis-Ojeda et al. (2014) and metallicities from new high-resolution spectroscopy by Petigura et al. (2017). Section 2 describes our observations and sample selection. Section 3 compares the metallicity distributions of the host stars of hot Jupiters, sub-Neptunes, and USP planets. Section 5 compares our results to those of Mulders et al. (2016). Section 6 provides some concluding remarks.

\section{Observations and Sample Selection}

Sanchis-Ojeda et al. (2014, hereafter SO+14) presented a catalog of USP planet candidates. We performed highresolution optical spectroscopy of 71 of the stars in this sample with the Keck I telescope and HIRES (Vogt et al. 1994) as part of the larger California Kepler Survey (CKS; Petigura et al. 2017). All of the stars brighter than $m_{\text {Kep }}=15.3$ were observed. Some fainter stars were also observed, particularly those hosting the planets with the shortest orbital periods. The spectra were collected from 2013 June to 2014 September. We used the standard California Planet Search setup, but without the iodine cell, giving a typical spectral resolution of $R=60,000$ over the wavelength range of $0.36-0.80 \mu \mathrm{m}$. The exposure times were typically 10 minutes, with a maximum exposure time of 20 minutes. For stars brighter than $m_{\mathrm{Kep}}=14.3$, we achieved a signal-to-noise ratio $(\mathrm{S} / \mathrm{N})$ of $40 \mathrm{pixel}^{-1}$ at $0.55 \mu \mathrm{m}$. For fainter stars, the $\mathrm{S} / \mathrm{N}$ was between 20 and 40 pixel $^{-1}$.

The spectroscopic parameters of each star were determined with a combination of SpecMatch, a template-matching code, and a variant of Spectroscopy Made Easy, a spectral synthesis code. Details are provided by Petigura et al. (2017), who demonstrated a precision of $60 \mathrm{~K}$ in effective temperature, 0.07 dex in surface gravity, and $0.04 \mathrm{dex}$ in $[\mathrm{Fe} / \mathrm{H}]$.

For our study we omitted stars with $T_{\text {eff }}<4700 \mathrm{~K}$. There are severe discrepancies between the synthesized and observed spectra for such cool stars, due to the onset of molecular absorption that is poorly treated in the Coelho et al. (2005) models. We also removed KOI 2813 and KIC 5955905, for which the apparent transit signals have been shown to be caused by binary stars rather than transiting planets. ${ }^{9}$

The mass and radius of each star were determined by Johnson et al. (2017), based on the comparison of the observed spectroscopic parameters with those calculated with the Dartmouth Stellar Evolution Program (Dotter et al. 2008), using the isochrones code (Morton et al. 2016). ${ }^{10}$ The inputs were $T_{\text {eff }}, \log g$, and $[\mathrm{Fe} / \mathrm{H}]$, along with their associated uncertainties. The code produces a posteriori distributions for the stellar mass, radius, and age, by interpolating between the available Dartmouth models. The radii of the transiting planets were then calculated from the stellar radii and the measured transit depths.

\footnotetext{
9 KOI 2813 was identified as a probable spectroscopic binary by Kolbl et al. (2015). KIC 5955905 is a probable background binary, based on observations of large chromatic variations in the apparent transit depth (E. Palle 2017, private communication).

${ }^{10}$ https://github.com/timothydmorton/isochrones (version 1.0).
}

\section{Metallicity Distributions}

We wanted to compare the metallicity distribution of the host stars of USP planets, hot Jupiters, and non-giant planets with periods longer than one day. To construct the appropriate samples we drew on the preceding results for the stars with USP planets, as well as the rest of the stars in the California Kepler Survey (Petigura et al. 2017). The larger sample includes about 1000 stars selected from the list of Kepler objects of interest (KOI), spanning a wide range of stellar types, planet sizes, and orbital periods. The stars were selected for spectroscopy independently of metallicity. Indeed, little information was available about the metallicities prior to the observations.

We restricted our attention to main-sequence stars with effective temperatures between 4700 and $6000 \mathrm{~K}$, the range encompassing almost all of the stars with USP planets. We constructed three samples:

1. USP planets: stars having a planet with an orbital period shorter than 1 day, selected from $\mathrm{SO}+14$ as described above. This sample has 64 stars.

2. Hot Jupiters: stars with a planet larger than $4 R_{\oplus}$ and an orbital period shorter than 10 days. The somewhat arbitrary value of $4 R_{\oplus}$ was chosen to match the value reported by Buchhave et al. (2012) and Buchhave et al. (2014) to distinguish different metallicity regimes. We omitted objects designated as "false positives" by Twicken et al. (2016) or Santerne et al. (2016). We also omitted objects with inferred sizes larger than $20 R_{\oplus}$ because experience has shown that in these cases the transit-like signal arises from a binary star rather than a transiting planet. This sample has 23 stars.

3. Hot small planets: stars with planets smaller than $4 R_{\oplus}$ and orbital periods between 1 and 10 days, after omitting objects designated as "false positives." This sample has 246 stars.

Tables 1 and 2 give the pertinent properties of the USP planets and hot Jupiters. Figure 1 shows the spectroscopic parameters $T_{\text {eff }}$ and $\log g$ for the stars in each sample. The dashed line is the boundary we used to identify main-sequence stars; our samples were restricted to stars below this line. Figure 2 shows the period-radius distribution of the planets hosted by the stars in each sample. In both figures, the small gray squares show the full sample of Kepler stars that were analyzed by Johnson et al. (2017).

Figure 3 focuses exclusively on the USP planets. All but one of the USP planets have sizes $\lesssim 2 R_{\oplus}$, even though no selection was made based on planet size. Thus, we confirm the finding of $\mathrm{SO}+14$ that USP planets are almost always smaller than $2 R_{\oplus}$. We find no major differences between our newly determined radius distribution and that of the distribution presented by $\mathrm{SO}$ +14 except that the new estimates of planetary radii have smaller uncertainties, and one of the outliers with size $>2 R_{\oplus}$ does not appear in the new sample. The single remaining USP planet with $R>2 R_{\oplus}$ is KOI 3913, a remarkable case which deserves further observation.

Figure 4 shows the distribution of $[\mathrm{Fe} / \mathrm{H}]$ for the stars in each sample. Even at a glance, the hot Jupiters appear to be weighted toward higher $[\mathrm{Fe} / \mathrm{H}]$ than either the USP planets or the hot small planets. The distributions for the USP and hot small planets appear similar to one another. To quantify these impressions we performed two-sample Kolmogorov-Smirnov 
Table 1

Characteristics of the USP Planet Sample

\begin{tabular}{|c|c|c|c|c|c|c|c|}
\hline ID & $T_{\text {eff }}[\mathrm{K}]$ & $\log g$ & {$[\mathrm{Fe} / \mathrm{H}]$} & $R_{\star}\left[R_{\odot}\right]$ & $M_{\star}\left[M_{\odot}\right]$ & $R_{p}\left[R_{\oplus}\right]$ & $P_{\text {orb }}[\mathrm{hr}]$ \\
\hline KOI 0072 & $5599_{-65}^{+66}$ & $4.340_{-0.100}^{+0.080}$ & $-0.110_{-0.040}^{+0.040}$ & $1.060_{-0.090}^{+0.160}$ & $0.910_{-0.030}^{+0.040}$ & $1.46_{-0.17}^{+0.17}$ & 20.2 \\
\hline KOI 0191 & $5459_{-64}^{+63}$ & $4.450_{-0.080}^{+0.070}$ & $0.100_{-0.040}^{+0.040}$ & $0.940_{-0.060}^{+0.090}$ & $0.920_{-0.030}^{+0.030}$ & $1.35_{-0.12}^{+0.12}$ & 17.0 \\
\hline KOI 0577 & $5085_{-66}^{+64}$ & $4.530_{-0.040}^{+0.040}$ & $0.110_{-0.040}^{+0.040}$ & $0.820_{-0.040}^{+0.040}$ & $0.830_{-0.030}^{+0.030}$ & $0.91_{-0.09}^{+0.09}$ & 15.4 \\
\hline KOI 0717 & $5619_{-68}^{+61}$ & $4.250_{-0.100}^{+0.090}$ & $0.310_{-0.040}^{+0.040}$ & $1.280_{-0.140}^{+0.170}$ & $1.050_{-0.040}^{+0.060}$ & $0.81_{-0.11}^{+0.11}$ & 21.6 \\
\hline KOI 1128 & $5352_{-63}^{+65}$ & $4.500_{-0.060}^{+0.060}$ & $-0.040_{-0.040}^{+0.040}$ & $0.870_{-0.050}^{+0.060}$ & $0.860_{-0.030}^{+0.030}$ & $1.24_{-0.10}^{+0.10}$ & 23.3 \\
\hline KOI 1150 & $5755_{-66}^{+68}$ & $4.320_{-0.090}^{+0.090}$ & $0.100_{-0.040}^{+0.040}$ & $1.150_{-0.110}^{+0.140}$ & $1.010_{-0.040}^{+0.040}$ & $1.02_{-0.12}^{+0.12}$ & 16.3 \\
\hline KOI 1169 & $5634_{-64}^{+66}$ & $4.360_{-0.090}^{+0.090}$ & $0.110_{-0.040}^{+0.040}$ & $1.080_{-0.100}^{+0.130}$ & $0.970_{-0.030}^{+0.030}$ & $1.52_{-0.16}^{+0.16}$ & 16.6 \\
\hline KOI 1239 & $5747_{-66}^{+66}$ & $4.380_{-0.080}^{+0.070}$ & $-0.040_{-0.040}^{+0.040}$ & $1.050_{-0.080}^{+0.110}$ & $0.970_{-0.030}^{+0.040}$ & $1.77_{-0.17}^{+0.17}$ & 18.7 \\
\hline KOI 1300 & $4764_{-65}^{+63}$ & $4.580_{-0.030}^{+0.030}$ & $0.030_{-0.040}^{+0.040}$ & $0.740_{-0.020}^{+0.020}$ & $0.760_{-0.020}^{+0.030}$ & $1.54_{-0.13}^{+0.13}$ & 15.1 \\
\hline KOI 1360 & $4960_{-64}^{+64}$ & $4.590_{-0.040}^{+0.030}$ & $-0.100_{-0.040}^{+0.040}$ & $0.740_{-0.020}^{+0.030}$ & $0.780_{-0.030}^{+0.030}$ & $0.87_{-0.09}^{+0.09}$ & 18.2 \\
\hline KOI 1367 & $4962_{-64}^{+64}$ & $4.590_{-0.040}^{+0.030}$ & $-0.080_{-0.040}^{+0.040}$ & $0.750_{-0.020}^{+0.030}$ & $0.780_{-0.030}^{+0.030}$ & $1.44_{-0.12}^{+0.12}$ & 13.7 \\
\hline KOI 1428 & $4776_{-65}^{+64}$ & $4.600_{-0.030}^{+0.030}$ & $-0.110_{-0.040}^{+0.040}$ & $0.710_{-0.020}^{+0.030}$ & $0.730_{-0.030}^{+0.030}$ & $1.90_{-0.21}^{+0.21}$ & 22.3 \\
\hline KOI 1442 & $5568_{-70}^{+58}$ & $4.260_{-0.100}^{+0.090}$ & $0.390_{-0.040}^{+0.040}$ & $1.260_{-0.140}^{+0.160}$ & $1.050_{-0.040}^{+0.060}$ & $1.43_{-0.18}^{+0.18}$ & 16.1 \\
\hline KOI 1655 & $5536_{-65}^{+64}$ & $4.450_{-0.070}^{+0.070}$ & $-0.070_{-0.040}^{+0.040}$ & $0.940_{-0.060}^{+0.080}$ & $0.900_{-0.030}^{+0.030}$ & $1.40_{-0.12}^{+0.12}$ & 22.6 \\
\hline KOI 1688 & $5979_{-64}^{+71}$ & $4.100_{-0.100}^{+0.100}$ & $0.170_{-0.040}^{+0.040}$ & $1.620_{-0.210}^{+0.290}$ & $1.220_{-0.080}^{+0.120}$ & $1.69_{-0.27}^{+0.27}$ & 22.1 \\
\hline KOI 1875 & $5576_{-64}^{+65}$ & $4.400_{-0.080}^{+0.070}$ & $-0.110_{-0.040}^{+0.040}$ & $0.980_{-0.080}^{+0.100}$ & $0.900_{-0.030}^{+0.030}$ & $1.38_{-0.13}^{+0.13}$ & 13.0 \\
\hline KOI 2039 & $5575_{-64}^{+64}$ & $4.490_{-0.060}^{+0.030}$ & $0.250_{-0.040}^{+0.040}$ & $0.950_{-0.040}^{+0.060}$ & $1.010_{-0.040}^{+0.030}$ & $0.81_{-0.08}^{+0.08}$ & 18.2 \\
\hline KOI 2079 & $5477_{-66}^{+68}$ & $4.380_{-0.090}^{+0.080}$ & $0.380_{-0.040}^{+0.040}$ & $1.070_{-0.090}^{+0.120}$ & $1.000_{-0.030}^{+0.040}$ & $0.75_{-0.08}^{+0.08}$ & 16.6 \\
\hline KOI 2093 & $5953_{-65}^{+66}$ & $4.350_{-0.080}^{+0.070}$ & $0.020_{-0.040}^{+0.040}$ & $1.140_{-0.100}^{+0.130}$ & $1.070_{-0.040}^{+0.040}$ & $1.34_{-0.15}^{+0.15}$ & 23.8 \\
\hline KOI 2119 & $5136_{-65}^{+65}$ & $4.540_{-0.050}^{+0.040}$ & $0.180_{-0.040}^{+0.040}$ & $0.830_{-0.030}^{+0.040}$ & $0.870_{-0.030}^{+0.030}$ & $1.32_{-0.11}^{+0.11}$ & 13.7 \\
\hline KOI 2202 & $5307_{-65}^{+64}$ & $4.470_{-0.070}^{+0.060}$ & $0.320_{-0.040}^{+0.040}$ & $0.930_{-0.050}^{+0.080}$ & $0.940_{-0.030}^{+0.030}$ & $1.22_{-0.11}^{+0.11}$ & 19.4 \\
\hline KOI 2248 & $5149_{-64}^{+65}$ & $4.540_{-0.050}^{+0.040}$ & $0.110_{-0.040}^{+0.040}$ & $0.830_{-0.030}^{+0.040}$ & $0.850_{-0.030}^{+0.030}$ & $1.15_{-0.10}^{+0.10}$ & 18.2 \\
\hline KOI 2250 & $4958_{-66}^{+65}$ & $4.570_{-0.040}^{+0.030}$ & $0.120_{-0.040}^{+0.040}$ & $0.780_{-0.030}^{+0.030}$ & $0.820_{-0.030}^{+0.030}$ & $1.62_{-0.14}^{+0.14}$ & 15.1 \\
\hline KOI 2281 & $5080_{-65}^{+65}$ & $4.530_{-0.040}^{+0.040}$ & $0.160_{-0.040}^{+0.040}$ & $0.820_{-0.040}^{+0.040}$ & $0.840_{-0.030}^{+0.030}$ & $0.90_{-0.16}^{+0.16}$ & 18.5 \\
\hline KOI 2393 & $4836_{-65}^{+65}$ & $4.590_{-0.040}^{+0.030}$ & $-0.060_{-0.040}^{+0.040}$ & $0.730_{-0.020}^{+0.030}$ & $0.760_{-0.030}^{+0.030}$ & $1.15_{-0.10}^{+0.10}$ & 18.5 \\
\hline KOI 2396 & $5228_{-65}^{+66}$ & $4.540_{-0.060}^{+0.040}$ & $0.100_{-0.040}^{+0.040}$ & $0.830_{-0.030}^{+0.050}$ & $0.870_{-0.030}^{+0.030}$ & $1.68_{-0.17}^{+0.17}$ & 12.0 \\
\hline KOI 2409 & $4774_{-66}^{+65}$ & $4.660_{-0.020}^{+0.030}$ & $-0.590_{-0.040}^{+0.040}$ & $0.620_{-0.020}^{+0.020}$ & $0.630_{-0.020}^{+0.020}$ & $1.26_{-0.10}^{+0.10}$ & 13.9 \\
\hline KOI 2492 & $5635_{-60}^{+62}$ & $4.370_{-0.090}^{+0.080}$ & $-0.310_{-0.040}^{+0.040}$ & $0.980_{-0.090}^{+0.110}$ & $0.830_{-0.030}^{+0.030}$ & $0.89_{-0.10}^{+0.10}$ & 23.5 \\
\hline KOI 2517 & $5601_{-64}^{+64}$ & $4.520_{-0.060}^{+0.030}$ & $-0.070_{-0.040}^{+0.040}$ & $0.880_{-0.040}^{+0.050}$ & $0.940_{-0.030}^{+0.030}$ & $1.01_{-0.09}^{+0.09}$ & 23.3 \\
\hline KOI 2571 & $5269_{-66}^{+65}$ & $4.480_{-0.070}^{+0.060}$ & $0.250_{-0.040}^{+0.040}$ & $0.900_{-0.050}^{+0.070}$ & $0.910_{-0.030}^{+0.040}$ & $1.06_{-0.09}^{+0.09}$ & 19.9 \\
\hline KOI 2607 & $5774_{-65}^{+63}$ & $4.400_{-0.090}^{+0.070}$ & $0.200_{-0.040}^{+0.040}$ & $1.070_{-0.080}^{+0.120}$ & $1.050_{-0.040}^{+0.040}$ & $1.78_{-0.19}^{+0.19}$ & 18.0 \\
\hline KOI 2668 & $5460_{-66}^{+66}$ & $4.510_{-0.060}^{+0.050}$ & $-0.040_{-0.040}^{+0.040}$ & $0.870_{-0.040}^{+0.060}$ & $0.900_{-0.030}^{+0.030}$ & $1.40_{-0.12}^{+0.12}$ & 16.3 \\
\hline KOI 2694 & $4787_{-66}^{+65}$ & $4.560_{-0.030}^{+0.030}$ & $0.230_{-0.040}^{+0.040}$ & $0.770_{-0.030}^{+0.030}$ & $0.790_{-0.030}^{+0.030}$ & $1.42_{-0.12}^{+0.12}$ & 20.2 \\
\hline KOI 2753 & $5840_{-63}^{+55}$ & $4.170_{-0.100}^{+0.100}$ & $0.210_{-0.040}^{+0.040}$ & $1.450_{-0.190}^{+0.230}$ & $1.130_{-0.060}^{+0.100}$ & $1.22_{-0.18}^{+0.18}$ & 22.6 \\
\hline KOI 2756 & $5904_{-61}^{+66}$ & $4.380_{-0.090}^{+0.070}$ & $0.100_{-0.040}^{+0.040}$ & $1.100_{-0.090}^{+0.130}$ & $1.070_{-0.040}^{+0.040}$ & $1.16_{-0.12}^{+0.12}$ & 16.1 \\
\hline KOI 2763 & $4727_{-65}^{+65}$ & $4.600_{-0.030}^{+0.030}$ & $-0.010_{-0.040}^{+0.040}$ & $0.720_{-0.020}^{+0.020}$ & $0.750_{-0.030}^{+0.030}$ & $1.14_{-0.11}^{+0.11}$ & 12.0 \\
\hline KOI 2796 & $5686_{-65}^{+68}$ & $4.350_{-0.100}^{+0.080}$ & $0.000_{-0.040}^{+0.040}$ & $1.080_{-0.100}^{+0.140}$ & $0.960_{-0.030}^{+0.030}$ & $1.09_{-0.13}^{+0.13}$ & 13.0 \\
\hline KOI 2874 & $5243_{-65}^{+64}$ & $4.510_{-0.050}^{+0.060}$ & $-0.080_{-0.040}^{+0.040}$ & $0.840_{-0.050}^{+0.050}$ & $0.820_{-0.030}^{+0.030}$ & $1.11_{-0.10}^{+0.10}$ & 8.4 \\
\hline KOI 2875 & $4967_{-64}^{+63}$ & $4.580_{-0.040}^{+0.030}$ & $-0.090_{-0.040}^{+0.040}$ & $0.750_{-0.030}^{+0.030}$ & $0.770_{-0.030}^{+0.030}$ & $1.44_{-0.12}^{+0.12}$ & 7.2 \\
\hline KOI 2879 & $5472_{-66}^{+65}$ & $4.510_{-0.060}^{+0.040}$ & $-0.010_{-0.040}^{+0.040}$ & $0.880_{-0.040}^{+0.060}$ & $0.910_{-0.030}^{+0.030}$ & $0.63_{-0.05}^{+0.05}$ & 8.2 \\
\hline KOI 2916 & $4978_{-65}^{+65}$ & $4.560_{-0.040}^{+0.040}$ & $-0.000_{-0.040}^{+0.040}$ & $0.770_{-0.030}^{+0.030}$ & $0.800_{-0.030}^{+0.030}$ & $1.00_{-0.10}^{+0.10}$ & 7.4 \\
\hline KOI 3009 & $5110_{-64}^{+65}$ & $4.550_{-0.050}^{+0.040}$ & $0.170_{-0.040}^{+0.040}$ & $0.820_{-0.030}^{+0.040}$ & $0.860_{-0.030}^{+0.030}$ & $1.04_{-0.10}^{+0.10}$ & 18.2 \\
\hline KOI 3032 & $5213_{-65}^{+64}$ & $4.430_{-0.060}^{+0.060}$ & $0.360_{-0.040}^{+0.040}$ & $0.950_{-0.060}^{+0.080}$ & $0.900_{-0.030}^{+0.030}$ & $1.43_{-0.14}^{+0.14}$ & 15.4 \\
\hline KOI 3065 & $5713_{-64}^{+63}$ & $4.480_{-0.070}^{+0.040}$ & $-0.000_{-0.040}^{+0.040}$ & $0.940_{-0.050}^{+0.070}$ & $0.980_{-0.030}^{+0.030}$ & $1.17_{-0.12}^{+0.12}$ & 21.6 \\
\hline KOI 3246 & $4847_{-66}^{+66}$ & $4.580_{-0.040}^{+0.030}$ & $0.130_{-0.040}^{+0.040}$ & $0.760_{-0.020}^{+0.030}$ & $0.800_{-0.030}^{+0.030}$ & $0.81_{-0.07}^{+0.07}$ & 16.6 \\
\hline KOI 3867 & $5566_{-63}^{+67}$ & $4.420_{-0.090}^{+0.070}$ & $0.140_{-0.040}^{+0.040}$ & $0.990_{-0.070}^{+0.100}$ & $0.960_{-0.030}^{+0.030}$ & $1.67_{-0.15}^{+0.15}$ & 22.6 \\
\hline KOI 3913 & $5952_{-63}^{+65}$ & $4.260_{-0.100}^{+0.090}$ & $0.180_{-0.040}^{+0.040}$ & $1.310_{-0.150}^{+0.190}$ & $1.140_{-0.050}^{+0.060}$ & $3.27_{-0.43}^{+0.43}$ & 13.9 \\
\hline KOI 4002 & $5207_{-64}^{+64}$ & $4.530_{-0.060}^{+0.040}$ & $0.200_{-0.040}^{+0.040}$ & $0.850_{-0.040}^{+0.050}$ & $0.890_{-0.030}^{+0.030}$ & $1.29_{-0.12}^{+0.12}$ & 12.5 \\
\hline KOI 4018 & $5479_{-65}^{+64}$ & $4.520_{-0.060}^{+0.040}$ & $-0.010_{-0.040}^{+0.040}$ & $0.870_{-0.040}^{+0.050}$ & $0.920_{-0.030}^{+0.030}$ & $1.28_{-0.11}^{+0.11}$ & 20.9 \\
\hline KOI 4070 & $4926_{-65}^{+66}$ & $4.570_{-0.040}^{+0.030}$ & $0.070_{-0.040}^{+0.040}$ & $0.770_{-0.030}^{+0.030}$ & $0.800_{-0.030}^{+0.030}$ & $1.09_{-0.10}^{+0.10}$ & 19.0 \\
\hline KOI 4072 & $5840_{-65}^{+61}$ & $4.260_{-0.100}^{+0.100}$ & $0.110_{-0.040}^{+0.040}$ & $1.270_{-0.140}^{+0.170}$ & $1.060_{-0.040}^{+0.050}$ & $1.02_{-0.13}^{+0.13}$ & 16.6 \\
\hline KOI 4109 & $4995_{-66}^{+66}$ & $4.530_{-0.040}^{+0.040}$ & $0.250_{-0.040}^{+0.040}$ & $0.830_{-0.040}^{+0.040}$ & $0.840_{-0.030}^{+0.030}$ & $0.72_{-0.07}^{+0.07}$ & 15.8 \\
\hline KOI 4144 & $6000_{-64}^{+65}$ & $4.390_{-0.080}^{+0.070}$ & $-0.110_{-0.040}^{+0.040}$ & $1.080_{-0.080}^{+0.110}$ & $1.030_{-0.040}^{+0.040}$ & $1.19_{-0.12}^{+0.12}$ & 23.5 \\
\hline KOI 4159 & $5233_{-66}^{+66}$ & $4.490_{-0.050}^{+0.060}$ & $0.120_{-0.040}^{+0.040}$ & $0.880_{-0.050}^{+0.050}$ & $0.860_{-0.030}^{+0.030}$ & $0.75_{-0.07}^{+0.07}$ & 23.3 \\
\hline KOI 4199 & $5109_{-65}^{+65}$ & $4.570_{-0.050}^{+0.030}$ & $-0.120_{-0.040}^{+0.040}$ & $0.770_{-0.030}^{+0.040}$ & $0.800_{-0.030}^{+0.030}$ & $0.77_{-0.07}^{+0.07}$ & 13.0 \\
\hline KOI 4366 & $5269_{-63}^{+66}$ & $4.530_{-0.060}^{+0.050}$ & $-0.110_{-0.040}^{+0.040}$ & $0.820_{-0.040}^{+0.050}$ & $0.830_{-0.030}^{+0.030}$ & $1.23_{-0.12}^{+0.12}$ & 18.2 \\
\hline KOI 4430 & $5104_{-66}^{+65}$ & $4.550_{-0.050}^{+0.040}$ & $0.100_{-0.040}^{+0.040}$ & $0.810_{-0.030}^{+0.040}$ & $0.840_{-0.030}^{+0.030}$ & $1.38_{-0.20}^{+0.20}$ & 12.2 \\
\hline KOI 4441 & $4888_{-65}^{+65}$ & $4.570_{-0.030}^{+0.030}$ & $0.060_{-0.040}^{+0.040}$ & $0.760_{-0.030}^{+0.030}$ & $0.790_{-0.030}^{+0.030}$ & $1.41_{-0.18}^{+0.18}$ & 16.3 \\
\hline
\end{tabular}


Table 1

(Continued)

\begin{tabular}{|c|c|c|c|c|c|c|c|}
\hline ID & $T_{\text {eff }}[\mathrm{K}]$ & $\log g$ & {$[\mathrm{Fe} / \mathrm{H}]$} & $R_{\star}\left[R_{\odot}\right]$ & $M_{\star}\left[M_{\odot}\right]$ & $R_{p}\left[R_{\oplus}\right]$ & $P_{\text {orb }}[\mathrm{hr}]$ \\
\hline KOI 4469 & $4909_{-64}^{+65}$ & $4.570_{-0.040}^{+0.030}$ & $0.070_{-0.040}^{+0.040}$ & $0.770_{-0.030}^{+0.030}$ & $\begin{array}{c}0.800_{-0.030}^{+0.030} \\
\end{array}$ & $0.71_{-0.07}^{+0.07}$ & 21.4 \\
\hline KOI 4746 & $4948_{-66}^{+66}$ & $4.570_{-0.040}^{+0.030}$ & $0.080_{-0.040}^{+0.040}$ & $0.780_{-0.030}^{+0.030}$ & $0.810_{-0.030}^{+0.030}$ & $0.83_{-0.08}^{+0.08}$ & 23.5 \\
\hline KOI 4841 & $4803_{-65}^{+65}$ & $4.590_{-0.030}^{+0.030}$ & $-0.010_{-0.040}^{+0.040}$ & $0.730_{-0.020}^{+0.030}$ & $0.760_{-0.030}^{+0.030}$ & $1.35_{-0.13}^{+0.13}$ & 17.0 \\
\hline KIC 8435766 & $5060_{-64}^{+65}$ & $4.570_{-0.040}^{+0.030}$ & $0.000_{-0.040}^{+0.040}$ & $0.780_{-0.030}^{+0.030}$ & $0.820_{-0.030}^{+0.030}$ & $1.25_{-0.14}^{+0.14}$ & 8.5 \\
\hline KIC 11187332 & $5573_{-65}^{+66}$ & $4.430_{-0.070}^{+0.070}$ & $-0.090_{-0.040}^{+0.040}$ & $0.960_{-0.070}^{+0.090}$ & $0.900_{-0.030}^{+0.030}$ & $1.17_{-0.17}^{+0.17}$ & 7.3 \\
\hline KIC 2718885 & $5614_{-64}^{+64}$ & $4.370_{-0.090}^{+0.080}$ & $0.120_{-0.040}^{+0.040}$ & $1.060_{-0.090}^{+0.120}$ & $0.960_{-0.030}^{+0.030}$ & $1.12_{-0.19}^{+0.19}$ & 4.7 \\
\hline
\end{tabular}

tests, which estimate the probability, $p$, that two samples are drawn from the same distribution. The results, given in Table 3, indicate that it is very unlikely that the USP planets and hot Jupiters are drawn from the same distribution, while the USP planets and the hot smaller planets have distributions that are indistinguishable with the current data.

\section{Upper Bound on Hot-Jupiter Fraction}

Evidently, the stars with USP planets have a different metallicity distribution than those of stars with hot Jupiters. We placed an upper bound on the fraction $f$ of members in the USP planet sample that could have been drawn from the same distribution as the hot-Jupiter sample, using a Monte Carlo technique. We considered the range of $f$ from zero to unity. For each choice of $f$, we constructed a sample of 64 metallicities, matching the actual USP sample size. We randomly drew (with replacement) $N=[64 f]$ values from the hot-Jupiter sample and $64-N$ values from the USP sample, where $[x]$ indicates rounding to the nearest integer. We added Gaussian errors to each metallicity with a standard deviation of 0.04 dex. We then computed the probability $p$ that the simulated sample was drawn from the same underlying distribution as the hot-Jupiter sample, using a two-sided Kolmogorov-Smirnov test. We repeated this procedure $10^{3}$ times and recorded the mean $p$-value.

For high values of $f$, the simulated sample is drawn entirely from the USP planets and the $p$-values are $\sim 10^{-3}$ as described in the previous section. For values of $f$ approaching unity, the $p$-values are $\sim 1$ because the hot-Jupiter sample is being compared with itself. To determine an upper bound on $f$, we sought the value for which $p=0.0455$, corresponding to a nominal $2 \sigma$ level of confidence. The result is $f<0.36$, implying that no more than about half of the metallicities of the USP host stars could have been drawn from the same metallicity distribution as the hot-Jupiter hosts.

\section{Comparison with Mulders et al. (2016)}

Mulders et al. (2016) studied the relationship between orbital period and stellar metallicity for a sample of 665 Kepler planet candidates. They found the mean metallicity of stars with planets shorter than 10 days to be higher than that for stars with longer-period planets. This was true for all planet sizes, with the strongest effect $(+0.25 \pm 0.07 \mathrm{dex})$ seen for the smallest planets $\left(<1.7 R_{\oplus}\right)$.

Our study is concerned exclusively with planets with $P<10$ days. To compare our data with theirs, we note that their Figure 2 shows the mean metallicity to be nearly constant for periods ranging from 0.6 to 5 days, before decreasing by $\approx 0.1$ dex from 5 to 10 days. Our data do not display such a period dependence: the hosts of planets with periods $<5$ days and 5-10 days have the same mean metallicity to within 0.02 dex. A broader comparison between the CKS metallicity scale and that of Mulders et al. (2016) also shows significant differences, which will be examined as part of a forthcoming CKS paper led by E. Petigura.

We also note that our sample of ultra-short-period planets differs from that of Mulders et al. (2016). Our sample has 65 planets, while their sample has 8 planets, 3 of which (KOI 2813, 2717, and 3204) have host stars with effective temperatures outside the range of our study (4700-6000 K).

\section{Conclusions}

The metallicity distribution of the host stars of USP planets does not resemble the metallicity distribution of the host stars of hot Jupiters. In particular, the stars with USP planets show no evidence of an association with high metallicity, unlike stars with hot Jupiters. The USP hosts have a mean metallicity near the Sun's value, and are similar to that of the general planethosting population of Kepler stars.

This basic result is also obtained if we make some small changes to our sample definitions. If we require hot Jupiters to have radii larger than $7 R_{\oplus}$ instead of $4 R_{\oplus}$, then the sample size decreases from 23 to 15 . The metallicity distribution of their host stars remains distinguishable from that of the stars with USP planets, though with a reduced statistical significance $(p=0.01)$. We also tried requiring the hot small planets to have radii between 2 and $4 R_{\oplus}$, i.e., we omitted the smaller planets that are more likely to be rocky. This is a more direct test of an evolutionary connection between USP planets and gas-rich planets at slightly longer periods. This reduces the sample size from 246 to 82 . When tested against the metallicity distribution of the stars with USP planets, the $p$-value changes from 0.39 to 0.10 , which is still too large to be considered as evidence for a significant difference.

We interpret these results as an argument against any theory in which most of the USP planets are descended from hot Jupiters. In such a theory, the stars that are currently observed to have USP planets were once hosts to hot Jupiters, and their metallicity distribution should be the same as those stars currently observed to have hot Jupiters. The only way we see to escape this conclusion-which seems unlikely - is to hypothesize that the process that converts hot Jupiters into USP planets also systematically lowers the metallicity of the host star by $\Delta$ $[\mathrm{Fe} / \mathrm{H}] \approx-0.15$, so as to match the metallicity distribution of the hosts of smaller Kepler planets.

The possibility that USP planets represent the solid cores of former hot Jupiters has already been deemed unlikely on theoretical grounds, because of the difficulty of removing such a massive gaseous atmosphere. Murray-Clay et al. (2009) modeled the wind launched from a gaseous planet by a star's 
Table 2

Characteristics of the Hot Jupiter Sample

\begin{tabular}{|c|c|c|c|c|c|c|c|}
\hline ID & $T_{\text {eff }}[\mathrm{K}]$ & $\log g$ & {$[\mathrm{Fe} / \mathrm{H}]$} & $R_{\star}\left[R_{\odot}\right]$ & $M_{\star}\left[M_{\odot}\right]$ & $R_{p}\left[R_{\oplus}\right]$ & $P_{\text {orb }}[\mathrm{hr}]$ \\
\hline KOI 0001 & $5815_{-65}^{+66}$ & $4.390_{-0.090}^{+0.080}$ & $0.010_{-0.040}^{+0.040}$ & $1.060_{-0.090}^{+0.120}$ & $1.010_{-0.030}^{+0.030}$ & $14.32_{-1.42}^{+1.42}$ & 59.3 \\
\hline KOI 0003 & $4867_{-65}^{+66}$ & $4.540_{-0.030}^{+0.040}$ & $0.310_{-0.040}^{+0.040}$ & $0.810_{-0.030}^{+0.030}$ & $0.830_{-0.030}^{+0.030}$ & $5.11_{-0.41}^{+0.41}$ & 117.4 \\
\hline KOI 0007 & $5833_{-67}^{+60}$ & $4.120_{-0.100}^{+0.110}$ & $0.170_{-0.040}^{+0.040}$ & $1.530_{-0.200}^{+0.240}$ & $1.120_{-0.060}^{+0.100}$ & $4.13_{-0.60}^{+0.60}$ & 77.0 \\
\hline KOI 0017 & $5667_{-63}^{+58}$ & $4.170_{-0.100}^{+0.100}$ & $0.340_{-0.040}^{+0.040}$ & $1.450_{-0.180}^{+0.220}$ & $1.110_{-0.060}^{+0.100}$ & $15.04_{-2.10}^{+2.10}$ & 77.5 \\
\hline KOI 0022 & $5885_{-58}^{+61}$ & $4.210_{-0.100}^{+0.100}$ & $0.200_{-0.040}^{+0.040}$ & $1.380_{-0.180}^{+0.210}$ & $1.130_{-0.060}^{+0.090}$ & $14.20_{-2.02}^{+2.02}$ & 189.4 \\
\hline KOI 0063 & $5660_{-63}^{+64}$ & $4.490_{-0.050}^{+0.030}$ & $0.230_{-0.040}^{+0.040}$ & $0.960_{-0.040}^{+0.050}$ & $1.030_{-0.030}^{+0.030}$ & $6.09_{-0.49}^{+0.49}$ & 226.3 \\
\hline KOI 0135 & $5951_{-66}^{+68}$ & $4.210_{-0.120}^{+0.100}$ & $0.320_{-0.040}^{+0.040}$ & $1.450_{-0.190}^{+0.260}$ & $1.230_{-0.060}^{+0.090}$ & $13.03_{-2.05}^{+2.05}$ & 72.5 \\
\hline KOI 0141 & $5322_{-63}^{+65}$ & $4.430_{-0.070}^{+0.070}$ & $0.300_{-0.040}^{+0.040}$ & $0.970_{-0.070}^{+0.090}$ & $0.930_{-0.030}^{+0.030}$ & $5.68_{-0.53}^{+0.53}$ & 62.9 \\
\hline KOI 0186 & $5802_{-63}^{+62}$ & $4.350_{-0.090}^{+0.080}$ & $0.180_{-0.040}^{+0.0400}$ & $1.130_{-0.100}^{+0.140}$ & $1.050_{-0.040}^{+0.040}$ & $14.97_{-1.60}^{+1.60}$ & 77.8 \\
\hline KOI 0195 & $5535_{-66}^{+64}$ & $4.480_{-0.070}^{+0.060}$ & $-0.160_{-0.040}^{+0.040}$ & $0.890_{-0.060}^{+0.080}$ & $0.870_{-0.030}^{+0.030}$ & $11.56_{-0.93}^{+0.93}$ & 77.3 \\
\hline KOI 0201 & $5526_{-67}^{+69}$ & $4.240_{-0.100}^{+0.100}$ & $0.350_{-0.040}^{+0.040}$ & $1.260_{-0.140}^{+0.170}$ & $1.020_{-0.040}^{+0.050}$ & $10.93_{-1.35}^{+1.35}$ & 101.5 \\
\hline KOI 0203 & $5714_{-64}^{+64}$ & $4.440_{-0.070}^{+0.040}$ & $0.310_{-0.040}^{+0.040}$ & $1.030_{-0.050}^{+0.080}$ & $1.080_{-0.040}^{+0.030}$ & $15.05_{-1.20}^{+1.20}$ & 35.8 \\
\hline KOI 0800 & $5904_{-61}^{+63}$ & $4.250_{-0.100}^{+0.100}$ & $0.200_{-0.040}^{+0.040}$ & $1.310_{-0.150}^{+0.200}$ & $1.130_{-0.050}^{+0.070}$ & $4.45_{-0.63}^{+0.63}$ & 65.0 \\
\hline KOI 0889 & $5311_{-66}^{+63}$ & $4.480_{-0.070}^{+0.060}$ & $0.220_{-0.040}^{+0.040}$ & $0.910_{-0.050}^{+0.070}$ & $0.900_{-0.030}^{+0.040}$ & $11.86_{-0.95}^{+0.95}$ & 213.1 \\
\hline KOI 1779 & $5861_{-65}^{+63}$ & $4.420_{-0.060}^{+0.040}$ & $0.300_{-0.040}^{+0.040}$ & $1.080_{-0.060}^{+0.080}$ & $1.130_{-0.040}^{+0.040}$ & $4.35_{-0.35}^{+0.35}$ & 111.8 \\
\hline KOI 1800 & $5611_{-65}^{+65}$ & $4.510_{-0.050}^{+0.030}$ & $0.070_{-0.040}^{+0.040}$ & $0.910_{-0.030}^{+0.050}$ & $0.980_{-0.030}^{+0.030}$ & $6.29_{-0.55}^{+0.55}$ & 187.0 \\
\hline KOI 3689 & $5988_{-67}^{+68}$ & $4.190_{-0.110}^{+0.100}$ & $0.030_{-0.040}^{+0.040}$ & $1.410_{-0.170}^{+0.220}$ & $1.110_{-0.040}^{+0.060}$ & $14.08_{-1.98}^{+1.98}$ & 125.8 \\
\hline
\end{tabular}

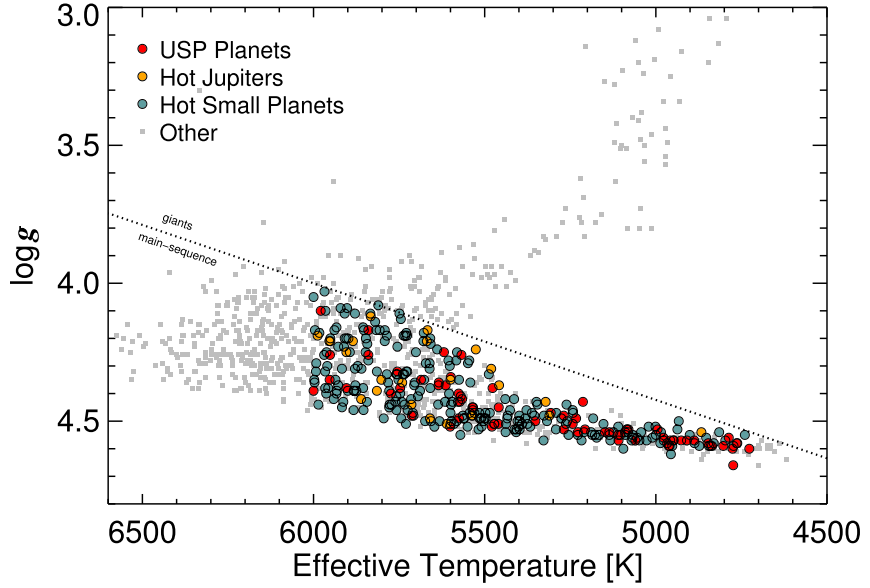

Figure 1. Spectroscopic parameters of the stellar samples. Stars below the dashed line were deemed main-sequence stars for the purpose of constructing our statistical samples, as described in Section 3. Colored circles show the parameters of the sample stars. The smaller squares are the broader sample of stars in the California Kepler Survey (Petigura et al. 2017).

high-energy radiation, and found it difficult to erode the entire atmosphere of a hot Jupiter. Had we found a strong metallicity enhancement for the hosts of USP planets, this theoretical conclusion would have been called into question.

It remains plausible that the progenitors of USP planets are Neptune-sized or smaller planets with gaseous atmospheres. This is also compatible with the tendency of USP planets to have sub-Neptune companions in somewhat wider orbits (Sanchis-Ojeda et al. 2014; Adams et al. 2017). Multiple theoretical studies have shown that it is possible to lose most of the gas from a low-density planet smaller than Neptune (Howe \& Burrows 2015; Ginzburg \& Sari 2016; Lopez 2016; Jackson et al. 2017). Also consistent with this picture is the recent

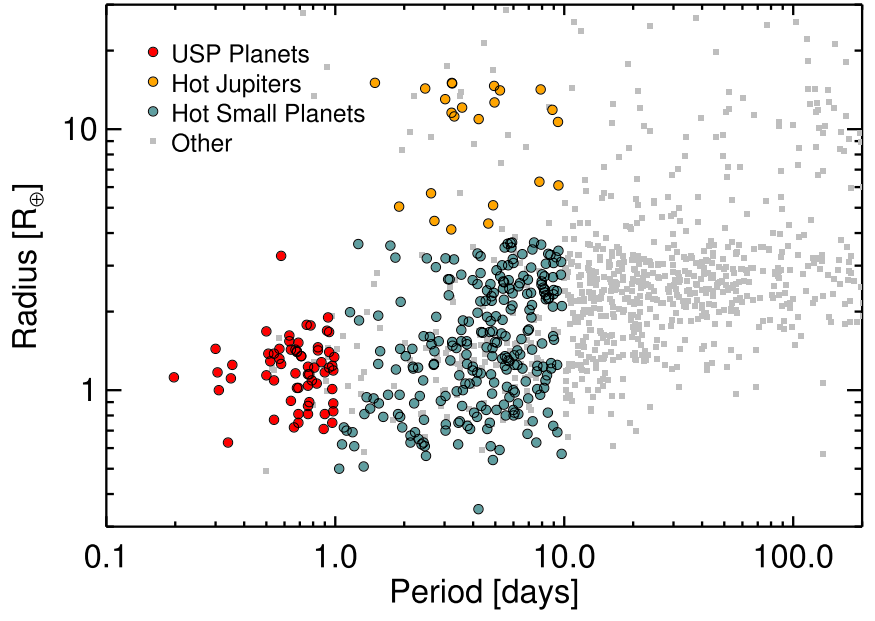

Figure 2. Orbital period and planetary radius. The colored circles show our statistical samples; the smaller squares are for the broader sample of stars in the California Kepler Survey (Petigura et al. 2017).

discovery by Fulton et al. (2017) that relatively few Kepler planets have sizes between 1.5 and $2 R_{\oplus}$. The missing planets in this size range might have been gas-rich sub-Neptunes whose atmospheres were stripped.

Ultra-short-period planets remain an attractive subject for future work to understand their origin, occurrence rate, radius distribution, and the dependence of all these quantities on the properties of the host star. The current sample of $\sim 100$ stars with USP planets have apparent magnitudes that are generally too faint for precise Doppler monitoring, observations of the Rossiter-McLaughlin effect, and detections of occultations or transmission effects. The TESS mission (Ricker et al. 2015) will help to remedy this problem by searching a similar number 


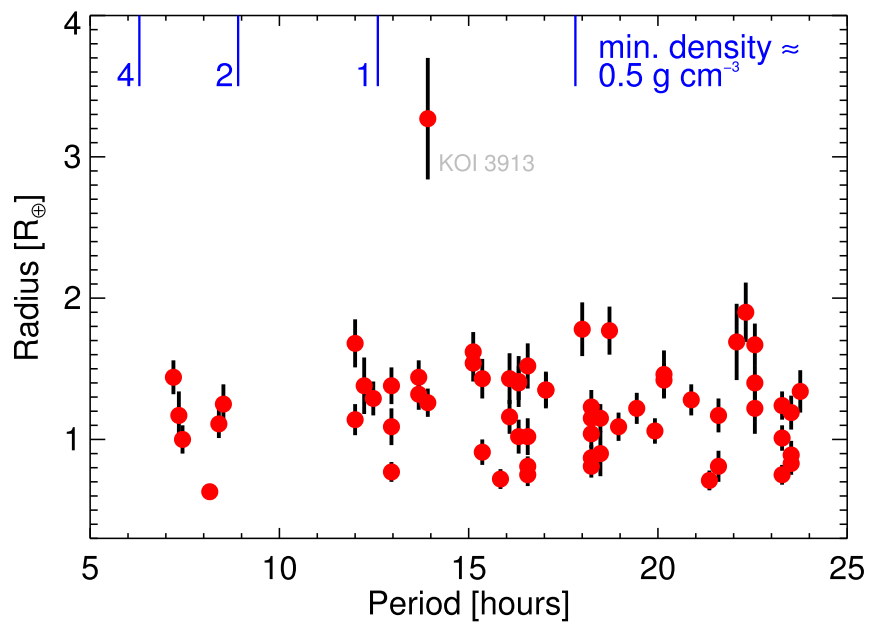

Figure 3. Orbital period and planetary radius for USP planets. Also marked are the Roche-limiting minimum periods for incompressible fluid bodies with mean densities of $0.5,1.0,2.0$, and $4.0 \mathrm{~g} \mathrm{~cm}^{-3}$, using Equation (2) of Rappaport et al. (2013). In reality, compression of the planetary interior may lower the minimum period by as much as $\approx 15 \%$.

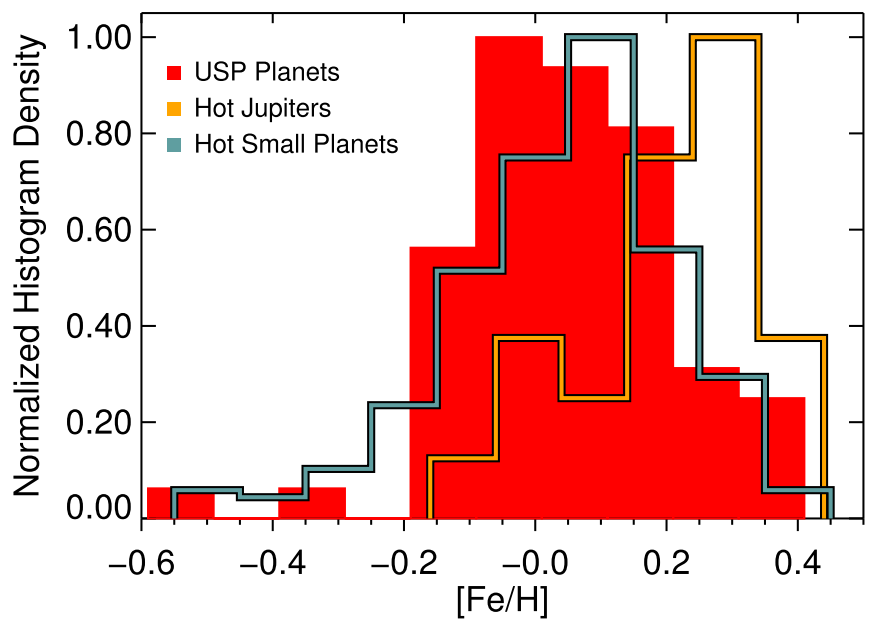

Figure 4. Metallicity distributions of the three statistical samples. The hotJupiter hosts have a different metallicity distribution (more weighted toward high metallicity) than the hosts of USP planets, and the hosts of planets smaller than Neptune with periods of 1-10 days.

Table 3

Comparisons between Metallicity Distributions

\begin{tabular}{lcccc}
\hline \hline \multirow{2}{*}{$\begin{array}{l}\text { Sample } \\
\text { name }\end{array}$} & \multirow{2}{*}{$\begin{array}{c}\text { Number } \\
\text { of stars }\end{array}$} & $\begin{array}{c}\text { Sample mean } \\
{[\mathrm{Fe} / \mathrm{H}]}\end{array}$ & \multicolumn{2}{c}{$p$ for comparison ${ }^{\mathrm{a}}$ with } \\
\cline { 5 - 6 } & 64 & $0.0584 \pm 0.0050$ & USPs & Hot Jupiters \\
\hline USP planets & 23 & $0.2096 \pm 0.0085$ & $3 \times 10^{-4}$ & $3 \times 10^{-4}$ \\
Hot Jupiters & 246 & $0.0459 \pm 0.0026$ & 0.39 & $2 \times 10^{-5}$ \\
\hline Hot small planets & 246 &
\end{tabular}

Note.

${ }^{a}$ Probability of being drawn from the same distribution, based on a two-sample Kolmogorov-Smirnov test.

of stars as the Kepler mission, but brighter by several magnitudes.

We thank the anonymous referee for a prompt and helpful report. We thank Simon Albrecht, Eugene Chiang, Brian Jackson, Eve Lee, Peter McCullough, Saul Rappaport, Amaury
Triaud, and Francesca Valsecchi for helpful discussions. We also thank Enric Palle for sharing his results for KIC 5955905. J.N.W. acknowledges the support from a NASA Keck PI Data Award, administered by the NASA Exoplanet Science Institute. A.W.H. acknowledges the support from NASA grant NNX12AJ23G. Data presented herein were obtained at the W.M. Keck Observatory from telescope time allocated to the National Aeronautics and Space Administration through the agency's scientific partnership with the California Institute of Technology and the University of California. The Observatory was made possible by the generous financial support of the W.M. Keck Foundation. The authors acknowledge the very significant cultural role and reverence that the summit of Mauna Kea has always had within the indigenous Hawaiian community. We are most fortunate to have the opportunity to conduct observations from this mountain.

\section{References}

Adams, E. R., Jackson, B., \& Endl, M. 2016, AJ, 152, 47

Adams, E. R., Jackson, B., Endl, M., et al. 2017, AJ, 153, 82

Adibekyan, V. Z., Delgado Mena, E., Sousa, S. G., et al. 2012, A\&A, 547, A36

Batalha, N. M., Borucki, W. J., Bryson, S. T., et al. 2011, ApJ, 729, 27

Becker, J. C., Vanderburg, A., Adams, F. C., Rappaport, S. A., \& Schwengeler, H. M. 2015, ApJL, 812, L18

Buchhave, L. A., Bizzarro, M., Latham, D. W., et al. 2014, Natur, 509, 593 Buchhave, L. A., \& Latham, D. W. 2015, ApJ, 808, 187

Buchhave, L. A., Latham, D. W., Johansen, A., et al. 2012, Natur, 486, 375 Chiang, E., \& Laughlin, G. 2013, MNRAS, 431, 3444

Coelho, P., Barbuy, B., Meléndez, J., Schiavon, R. P., \& Castilho, B. V. 2005, A\&A, 443, 735

Dawson, R. I., \& Fabrycky, D. C. 2010, ApJ, 722, 937

Demory, B.-O., Gillon, M., Deming, D., et al. 2011, A\&A, 533, A114

Dong, S., Zheng, Z., Zhu, Z., et al. 2014, ApJL, 789, L3

Dotter, D., Chaboyer, B., Jevremović, D., et al. 2008, ApJS, 178, 89

Fischer, D. A., \& Valenti, J. 2005, ApJ, 622, 1102

Fulton, B. J., Petigura, E. A., Howard, A. W., et al. 2017, arXiv:1703.10375

Ginzburg, S., \& Sari, R. 2017, MNRAS, 469, 278

Gonzalez, G. 1997, MNRAS, 285, 403

Guo, X., Johnson, J. A., Mann, A. W., et al. 2017, ApJ, 838, 25

Howe, A. R., \& Burrows, A. 2015, ApJ, 808, 150

Ida, S., \& Lin, D. N. C. 2004, ApJ, 616, 567

Jackson, B., Arras, P., Penev, K., Peacock, S., \& Marchant, P. 2017, ApJ, 835,145

Jackson, B., Stark, C. C., Adams, E. R., Chambers, J., \& Deming, D. 2013 , ApJ, 779, 165

Johnson, J. A., Petigura, E. A., Fulton, B. J., et al. 2017, arXiv:1703.10402

Kolbl, R., Marcy, G. W., Isaacson, H., \& Howard, A. W. 2015, AJ, 149, 18

Lee, E. J., \& Chiang, E. 2017, ApJ, 842, 40

Léger, A., Rouan, D., Schneider, J., et al. 2009, A\&A, 506, 287

Lopez, E. D. 2016, arXiv: 1610.01170

Lundkvist, M. S., Kjeldsen, H., Albrecht, S., et al. 2016, NatCo, 7, 11201

Mann, A. W., Gaidos, E., Kraus, A., \& Hilton, E. J. 2013, ApJ, 770, 43

Morton, T. D., Bryson, S. T., Coughlin, J. L., et al. 2016, ApJ, 822, 86

Mulders, G. D., Pascucci, I., Apai, D., Frasca, A., \& Molenda-Żakowicz, J. 2016, AJ, 152, 187

Murray-Clay, R. A., Chiang, E. I., \& Murray, N. 2009, ApJ, 693, 23

Petigura, E. A., Howard, A. W., Marcy, G. W., et al. 2017, arXiv:1703.10400 Rappaport, S., Sanchis-Ojeda, R., Rogers, L. A., Levine, A., \& Winn, J. N. 2013, ApJL, 773, L15

Ricker, G. R., Winn, J. N., Vanderspek, R., et al. 2015, JATIS, 1, 014003

Rogers, L. A. 2015, ApJ, 801, 41

Sanchis-Ojeda, R., Rappaport, S., Winn, J. N., et al. 2013, ApJ, 774, 54

Sanchis-Ojeda, R., Rappaport, S., Winn, J. N., et al. 2014, ApJ, 787, 47

Santerne, A., Moutou, C., Tsantaki, M., et al. 2016, A\&A, 587, A64

Santos, N. C., Israelian, G., \& Mayor, M. 2004, A\&A, 415, 1153

Schlaufman, K. C. 2015, ApJL, 799, L26

Schlaufman, K. C., \& Laughlin, G. 2011, ApJ, 738, 177

Schlaufman, K. C., Lin, D. N. C., \& Ida, S. 2010, ApJL, 724, L53

Sousa, S. G., Santos, N. C., Israelian, G., Mayor, M., \& Udry, S. 2011, A\&A, 533, A141

Steffen, J. H., \& Coughlin, J. L. 2016, PNAS, 113, 12023 
Terquem, C. 2014, MNRAS, 444, 1738

Twicken, J. D., Jenkins, J. M., Seader, S. E., et al. 2016, AJ, 152, 158

Udry, S., Mayor, M., Benz, W., et al. 2006, A\&A, 447, 361

Valsecchi, F., Rasio, F. A., \& Steffen, J. H. 2014, ApJL, 793, L3

Vanderburg, A., Bieryla, A., Duev, D. A., et al. 2016, ApJL, 829, L9
Vogt, S. S., Allen, S. L., Bigelow, B. C., et al. 1994, Proc. SPIE, 2198, 362 Wang, J., \& Fischer, D. A. 2015, AJ, 149, 14

Weiss, L. M., \& Marcy, G. W. 2014, ApJL, 783, L6

Winn, J. N., Matthews, J. M., Dawson, R. I., et al. 2011, ApJL, 737, L18

Zhu, W., Wang, J., \& Huang, C. 2016, ApJ, 832, 196 